ISSN: 2386-3919 - e-ISSN: 2386-3927

DOI: https://doi.org/10.14201/et20163426575

\title{
RELACIÓN ENTRE CREATIVIDAD Y LATERALIDAD EN EDUCACIÓN INFANTIL
}

\section{Relationship between creativity and laterality in Early Childhood Education}

\author{
Rocío Berenguer Sánchez, Fátima Llamas Salguero* \\ y Verónica López FERNÁNDEZ*** \\ Universidad Internacional de la Rioja. Facultad de Educación (UNIR) \\ *Correo-e: fatima.llamas@unir.net \\ ** Correo-e:veronica.lopez@unir.net
}

Recibido: 19/10/205; Aceptado: 15/9/2016; Publicado: 30/11/2016

Ref. Bibl. ROCÍO BERENGUER SÁNCHEZ, FÁTIMA LLAMAS SALGUERO y VERÓNICA LÓPEZ FERNÁNDEZ. Relación entre creatividad y lateralidad en Educación Infantil. Enseñanza \& Teaching, 34, 2-2016, 65-75.

RESUMEN: En Educación Infantil es fundamental conocer y desarrollar metodologías utilizando el desarrollo de la creatividad y la lateralidad en el proceso de enseñanza-aprendizaje en nuestros/as alumnos/as. Es un periodo idóneo para estudiar la relación existente entre estas variables. Por ello, entendemos la creatividad como una parte integral de todos los lenguajes en los que se expresan los niños/as en Educación Infantil (lenguaje verbal y escrito, plástico, corporal...), porque todos los lenguajes suponen un proceso creador, una forma de comunicarse con los demás, ya sea de una forma verbal, plástica o escrita. Todas esas formas de comunicarse se relacionan, además, con otro concepto como es la lateralidad. Es fundamental identificar y examinar la importancia de la lateralidad y las dominaciones en Educación Infantil ya que todos estos procesos son necesarios antes de acceder a otros lenguajes como la lectoescritura. El objetivo general es determinar la relación existente entre lateralidad y creatividad en niños/as de cuatro años de edad. Para ello, se han evaluado 60 niños del segundo ciclo de Educación Infantil de las variables creatividad y lateralidad definida y no 
definida. En el desarrollo de esta investigación se aplicó el test de Pensamiento Creativo de Torrance (1974) de expresión figurada y el test de lateralidad de la prueba neuropsicológica (2011). Los resultados obtenidos muestran que la mayoría de los alumnos/as tienen la lateralidad definida con un $75 \%$. Estos alumnos/ as obtienen mayores puntuaciones medias en cada componente de creatividad que el grupo con lateralidad no definida, así como una creatividad mayor que el grupo con lateralidad no definida.

Palabras clave: creatividad; lateralidad; educación infantil; innovación; formación; docentes.

SUMMARY: In primary education is essential to know and develop methodologies using the development of creativity and laterality in the process of teaching and learning in our student. It is an ideal place to study the relationship between these variables period. As we understand creativity as an integral part of all the languages in which student in early childhood education (verbal and written language, plastic body...) are expressed, for all languages represent a creative process, a way to communicate with others, either verbal or written form, plastic. All these forms of communication is also related to another concept as laterality. It is essential to identify and examine the importance of laterality and dominations in kindergarten because all these processes are required before accessing other languages such as literacy. The objective of this study is to describe the relationship between creativity and laterality in Early Childhood Education. This has been evaluated 60 children in the second cycle of Infant Education and creativity variables defined and undefined laterality. In the development of this research test Torrance Creative Thinking (1974) of figurative expression and the test of laterality of the neuropsychological test (2011) it was applied. The results show that most of the student have defined laterality with $75 \%$. These student earn higher average scores on each component of creativity, the group with undefined laterality and more creativity than the group with undefined laterality. teachers.

Key words: creativity; laterality; early childhood education; innovation; training

\section{INTRODUCCIÓN}

En la Educación Infantil los términos innovación, cambio metodológico, creatividad y lateralidad siempre deben estar presentes en la práctica educativa. La labor del docente va a estar centrada en la formación constante para poder aplicarlo en el proceso de enseñanza-aprendizaje (metodología, unidades didácticas, proyectos de trabajo, espacios y tiempos, actividades, etc.).

En este artículo se pretende relacionar los términos de creatividad y lateralidad ya que la creatividad forma parte de todos los lenguajes en los que se expresan los niños/as en Educación Infantil (lenguaje verbal y escrito, plástico, corporal...) y porque todos los lenguajes suponen un proceso creador, una forma de 
comunicarse con los demás, ya sea de una forma verbal, plástica o escrita. Todas esas formas de comunicarse se relacionan, además, con otro concepto a destacar, la lateralidad.

Ferré y Aribau (2002) identifica la importancia de examinar, en las escuelas, la lateralidad del niño para ver las dominancias de la mano (3-4 años), del ojo (a los 4-5 años) y del pie (a los 5-6 años). Cabe destacar que todos estos procesos son considerados de suma importancia ya que es necesario llevarlos a cabo antes de acceder a otros lenguajes como puede ser la lectoescritura, porque necesita una correcta orientación en el espacio (de izquierda a derecha y de arriba abajo).

Otras referencias son Martín Lobo (2003) y Ferré y Aribau (2002), que siguen la corriente de pensamiento encabezada por Portellano (2005) y aunque no deducen que tener una dominancia lateral establecida equivale a tener éxito académico, ni que lo contrario sea igual a tener dificultades de aprendizaje, sí que les parece que tener una lateralidad establecida significa que la información entra a nuestro cerebro de una forma ordenada y que genera una respuesta igualmente ordenada y eficaz.

Por todo ello, resaltamos que la creatividad y la lateralidad son necesarias para el desarrollo del niño, en la práctica del aula, pero también para su desarrollo social. En este sentido, Aluni y Penagos (2000) nos indican que la creatividad es fundamental y necesaria, indican que la historia siempre ha sido cambiada por este tipo de personas, personas que ven donde otros no.

Así, el objetivo general es determinar la relación existente entre lateralidad y creatividad en niños/as de cuatro años de edad. Conocer la posible relación entre los componentes de la creatividad (flexibilidad, fluidez, originalidad y elaboración) y la lateralidad definida y no definida y comprobar si los alumnos más creativos tienen una mejor dominancia lateral o, por el contrario, son dos variables que no están relacionadas.

Según la Enciclopedia de Psicopedagogía Océano (1998: 779-780), la creatividad es "La disposición a crear, que existe en estado potencial en todo individuo y a todas las edades». En otras líneas, para la mayoría de autores, como Sternberg y Lubart (1999), Mumford (2003) y Boden (2004), la creatividad es una forma de pensar, que da como resultado unos productos de cualquier naturaleza (artefactos, pintura, ideas...), que tienen a la vez originalidad (porque son nuevos) y valor (porque son útiles).

Otros autores, como Guilford (1952 y 1971), Thurstone (1952), Osborn (1953), Flanagan (1958) o Torrance (2000), parecen abundar en el concepto de creatividad anterior. Hacen referencia a una forma de pensar que da productos novedosos y que tienen valor.

Para Sternberg y Lubart (1997), hay seis factores que son los pilares básicos para el desarrollo de la creatividad, como la inteligencia, el conocimiento, los estilos de pensamiento, la personalidad, la motivación y el contexto medioambiental.

En otros términos, la neuropsicología de la creatividad se refiere a la evaluación de las capacidades creativas a través de pruebas como las de Regard y Landis (1988), que comprobaron que el hemisferio cerebral derecho era más creativo que el izquierdo. 
En el mismo sentido Montañes (1999) también considera al hemisferio derecho como el hemisferio creativo y al izquierdo como el hemisferio racional, sin embargo, para Puente Ferrerras, en su obra El cerebro creador (1999: 218), «... asignar la creatividad al hemisferio derecho, y la ciencia al hemisferio izquierdo, es incorrecto: porque implicaría que la actividad científica no necesita habilidades artísticas... y que éstas, nada tienen que ver con el quehacer científico».

En cambio, Bogousslavsky y Boller (2005) afirman que el cerebro participa como un todo en la creación artística, siendo éste un proceso que se realiza en tres etapas: procesamiento perceptual, extracción de rasgos y abstracción y, por último, la ejecución. Estos autores afirman que va a ser el área frontal la que juegue un papel crítico en la creatividad, tanto en funciones proactivas como inhibitorias, y que la desinhibición es necesaria para permitir el surgimiento de la novedad, que es prerrequisito de la creatividad. De igual forma, el investigador y profesor Degraff (1995) afirma que todos somos creativos, pero lo somos de distinta forma y en distinto grado. Considera que debemos practicar los cinco tipos de creatividad para aumentar todo nuestro potencial creador en creatividad mimética (consiste en tomar una idea de determinada área y aplicarla en otra), creatividad bisociativa (nos referimos a conectar una idea conocida con una idea desconocida para generar un concepto diferente), creatividad analógica (las analogías sirven para transferir información que entendemos con el fin de resolver desafíos en un área que desconocemos), creatividad narrativa (consiste en la capacidad de crear historias) y creatividad intuitiva (consiste en vaciar la mente, relajarse y crear un estado de conciencia fluido que permita a las ideas llegar con facilidad).

Dadamia (2001) clasifica los distintos periodos creativos del niño en: multisensorial, de 0 a 3 años, cuyo objetivo es despertar la curiosidad natural por el mundo que le rodea; simbólica, de 3 a 5 años, desarrollo de la capacidad de observar y la espontaneidad; intuitiva, de 5 a 7 años, desarrollo de la imaginación y la simbolización; creativa, de 7 a 10 años, cuyo fin es reinventar y reestructurar la realidad; operativa, de 10 a 15 años, desarrollo de la capacidad de formular hipótesis.

Cuando nos centramos en el término lateralidad, destacamos a Dorsch (1985), que considera la lateralidad como la dominancia lateral en la estructura y función de los órganos duplicados. Aparece con claridad en la mano, pero también se da en los ojos, oídos, brazos, piernas y pies. Portellano (2005) recoge distintas aproximaciones a destacar junto al concepto de lateralidad y nos habla de diferencia, asimetría, dominancia y lateralidad.

En cuanto a la diferencia, entiende que cada hemisferio tiene competencias cognitivas diferentes. Por asimetría cerebral, entiende que cada zona de un hemisferio puede procesar información de un tipo distinto, diferente a su zona homóloga del otro hemisferio. En relación a la dominancia, habla del predominio de un hemisferio sobre el otro, para cinco tipos de dominancia: ocular, referida, auditiva, pédica y manual. Autores como Ferré y Aribau (2002) utilizan el término referente en lugar de dominante. 
Y, por último, Portellano (2005) se refiere a la lateralidad como la preferencia a usar una de las partes simétricas del cuerpo más que la otra.

Los factores que influyen y que deben ser destacados en la lateralidad según Rizal (1987) son:

1. Factores Neurológicos, que explican dos teorías opuestas, una teoría que piensa que la lateralidad se ve determinada por la existencia de dos hemisferios cerebrales, produciéndose el dominio de uno frente al otro. Otra teoría afirma que todas las actividades han de ser realizadas por ambos hemisferios, no pudiendo determinarse la incidencia, o no, de este aspecto sobre la lateralidad.

2. Factores Sociales, podemos destacar la significación religiosa, por un lado, y por otro, el lenguaje. La significación religiosa hace alusión a la antigüedad cuando se forzaba el uso de la mano derecha, ya que la izquierda se consideraba un pacto con el diablo. Y el lenguaje, ya que, en el lenguaje escrito, la escritura se realiza de izquierda a derecha y en el lenguaje oral, el diestro se ha considerado siempre como algo bueno y el zurdo como algo siniestro.

3. Factores Ambientales, decir que la sociedad está organizada para los diestros, teniendo los zurdos muchas complicaciones para adaptarse a ella.

4. Factores Genéticos. Algunos estudios clínicos coinciden en que la preferencia lateral está determinada, en gran medida, por la herencia. Actualmente se tiende a pensar que la lateralidad viene determinada por un conjunto de todos los factores anteriormente expuesto.

Por todo ello, para la formación de docentes y el interés en este estudio, es fundamental conocer la neuropsicología de la lateralidad, que estudia la especialización de los hemisferios cerebrales. Aunque Paul Broca (1861) identificó la existencia de una asimetría cerebral, no será hasta finales del siglo xx cuando se demuestre que cada hemisferio realiza funciones muy particulares. El cerebro está formado por dos hemisferios aparentemente iguales, divididos a su vez en cinco lóbulos, comunicados entre sí y con el hemisferio contralateral. Cada lóbulo tiene unas funciones cerebrales diferentes:

- Lóbulo occipital, recoge toda la información visual.

- Lóbulo parietal, capta información táctil e integra la información sensorial y organiza el pensamiento.

- Lóbulo frontal: organiza el pensamiento y facilita el autocontrol.

- Lóbulo temporal, realiza funciones de audición, memoria, lenguaje e integración sensorial.

En cuanto al procesamiento de la información es el hemisferio izquierdo el que va a controlar el pensamiento racional y abstracto, mientras que el hemisferio derecho va a controlar más la parte emocional. 
Son estas diferencias las que van a dar lugar a la especialización de cada hemisferio, que tiene como resultado la lateralización, y que, en las funciones cerebrales complejas, es necesaria una actividad conjunta de los hemisferios a través del cuerpo calloso. Dado que la creatividad es una actividad compleja podemos afirmar que la lateralidad no definida supone un hándicap en el desarrollo de la creatividad.

Para Llamas (2003) se puede clasificar la lateralidad en cinco tipos, y sostiene que la dominancia de la extremidad superior se acompaña siempre de la preferencia idéntica ocular e incluso de la dominancia del mismo pie.

- Diestros: sujetos con predominio claro del lado derecho.

- Zurdos: sujetos con predominio claro del lado izquierdo.

- Lateralidad mixta (ambidiestros): sin predominio claro y usando indistintamente ambos lados del cuerpo. Zurdo para algunas actividades y diestro para otras.

- Lateralidad cruzada o heterogénea: predominio lateral derecho en unos miembros y zurdo en otros (p. ej. ojo derecho con mano izquierda).

- Zurdos contrariados: sujetos que por coacción familiar y/o escolar han sido adiestrados hacia el lado derecho.

Además establece la evolución de la lateralidad en cuatro periodos (Llamas, 2003):

- De 0-4 años. Percepción corporal global y descubrimiento de los lados corporales centrado en la mano. La dominancia lateral se afirma hacia los 4 años.

- De 4-6 años. Denominación de derecha-izquierda a las partes más utilizadas. Percepción del eje de simetría en objetos estáticos, proyectando en ellos la lateralidad propia.

- De 6-8 años. Denominación derecha-izquierda en los lados corporales del otro. Descubrimiento de lateralidad de objetos dinámicos. Cruce del eje de simetría imitando un compañero frente a sí.

- De 8-12 años. Proyección de derecha-izquierda en el espacio con el propio cuerpo como referencia u orientándose a partir de un punto del espacio (lectura e interpretación de planos, mapas).

Por todo ello, para relacionar la creatividad y la inteligencia son varios los estudios que ponen de manifiesto la importancia que tienen. Uno de los estudios más relevantes sobre creatividad y rendimiento escolar es el de Getzels y Jackson (1962) con dos grupos, el creativo y el inteligente, que alcanzaban los mismos niveles de rendimiento. Su conclusión fue que los «inteligentes» necesitan encontrar la respuesta correcta, mientras los "creativos" daban respuestas abiertas y nuevas al problema planteado. Torrance (1962), basándose en el estudio anterior, llegó a la misma conclusión, considerando a la creatividad casi independiente del CI. Es 
decir, a bajos niveles de CI, la creatividad está baja, pero a altos niveles de CI no se corresponden, necesariamente, altos niveles de creatividad.

En cuanto al papel de la lateralidad en el aprendizaje, hay que destacar el estudio de Claude (1978), que fue uno de los primeros en proponer la estimulación de la lateralidad en edad temprana. Entre sus conclusiones, está la relación entre los problemas de lateralidad y los problemas de aprendizaje. También Lesak y Crow (2001) en sus discursos destacan como ventaja tener una lateralidad definida.

A juzgar por los datos científicos anteriores, hay relación entre creatividad y rendimiento escolar y entre problemas de lateralidad y problemas de aprendizaje, pero ¿hay una posible relación entre lateralidad y creatividad?, ¿es posible vincular la capacidad creativa de los niños de 4 años con que tengan su lateralidad definida o no?

\section{Metodología}

El diseño es el de una investigación descriptiva, no experimental, en el que se busca conocer una situación concreta, en este caso, conocer la lateralidad definida y no definida y la creatividad de un grupo de niños y su posible relación. Por todo ello, esta investigación cuenta con el siguiente objetivo general: determinar la relación existente entre lateralidad y creatividad en niños/as de cuatro años de edad.

La muestra estuvo compuesta por 60 niños/as del 2. ${ }^{\circ}$ nivel del $2 .^{\circ}$ ciclo de Educación Infantil (4-5 años de edad), de dos colegios públicos de Infantil y Primaria de línea dos, de la ciudad de Valladolid. Todos fueron evaluados, previo consentimiento informado de los padres, tutores y Equipo Directivo. Las variables medidas son la dependiente creatividad, a través de sus cuatro componentes, según Torrance (1974): flexibilidad, fluidez, originalidad y elaboración, siendo la creatividad global la suma aritmética de los diferentes componentes y la variable lateralidad, entendida como la predominancia lateral visual, manual, auditiva y pédica en el alumnado, lo que determinará su lateralidad global.

Los instrumentos aplicados son:

Para evaluar la creatividad se ha empleado el test de pensamiento creativo de Torrance: expresión figurada (1974). Su finalidad es evaluar el nivel de creatividad realizando dibujos, valorando los componentes de originalidad, fluidez, flexibilidad y elaboración. Está formado por tres juegos que el alumno debe desarrollar:

- Componer un dibujo: se le pide que construya un dibujo a partir de una forma dada en papel de color verde, que podría parecerse a un huevo. Se miden los componentes originalidad y elaboración con valores de 0 a 5 puntos.

- Acabar un dibujo: el niño tiene que completar las 10 figuras incompletas que se dan. Se miden originalidad y elaboración con valores de 0 a 50 puntos y de 0 a 10 puntos para fluidez y flexibilidad.

- Componer diferentes realizaciones utilizando líneas paralelas. El niño tiene que hacer tantos dibujos como pueda con treinta pares de líneas paralelas. 
Se miden originalidad y elaboración con valores de 0 a 150 puntos y de 0 a 30 para fluidez y flexibilidad.

Para evaluar la lateralidad se ha empleado el test de lateralidad de la prueba neuropsicológica (adaptado por Martín Lobo, G. ${ }^{a}$ Castellón, Rodríguez y Vallejo, 2011) del equipo del Instituto de Neuropsicología y Educación, Fomento. Presenta como finalidad evaluar la lateralidad en los niños a partir de 4 años. Compuesto por cuatro pruebas que nos van a permitir medir la lateralidad a nivel visual, auditivo, manual y pédica. Cada una, a su vez, se subdivide en 10 pruebas. Según van realizando cada prueba, se marca con una cruz el lado del cuerpo que utilizan (diestro o zurdo) y, con estos datos, se saca el tipo de lateralidad que el niño tiene en cada uno de los niveles. Para determinar la lateralidad, se tiene en cuenta que en cada prueba se obtengan más de 6 ítems en un mismo lado: derecho o izquierdo. Si tiene menos de 6 ítems en un mismo lado supone tener lateralidad sin definir.

Los materiales empleados para aplicar la prueba fueron: tubos grandes y pequeños, marionetas de dedo, tijeras, reloj, cajas de cartón, llave, forma de borrar, pelota y peine.

El procedimiento llevado a cabo se realizó administrando las dos pruebas en diferentes días para no cansar a los alumnos/as. La prueba de lateralidad se aplicó individualmente y se hizo en primer lugar. Con los test realizados, se hace la corrección de los mismos y se codifican las puntuaciones para cada sujeto.

La prueba de creatividad se aplicó colectivamente, en cuatro sesiones, una por aula.

El estudio se ha realizado con el programa spss 2013. A partir de los datos obtenidos, realizamos el análisis descriptivo de los resultados obtenidos. Estos datos descriptivos se exponen en forma de frecuencias y porcentajes para lateralidad, y en forma de medias, desviación típica y coeficiente de variación para creatividad, para mostrar de forma simplificada los datos conseguidos.

\section{RESUlTADOS Y DisCUSIÓN}

El análisis de resultados muestra, en la Tabla 1, el mayoritario porcentaje de niños con lateralidad definida (totalmente diestros y/o zurdos en ojo, ó́do, mano y pie): el $75 \%$ frente al 15\% que la tienen sin definir.

Se confirma la predominancia óculo-manual pédica (3-6 años) demostrada por Ferré y Ariabu (2002) aunque el estudio halla la predominancia pédica en la franja de edad 4-5 años, un año antes (5-6 años) que el autor citado.

Pueden haber influido factores sociales y ambientales, aludidos por Rigal (1987), en este porcentaje mayoritario, y que el estudio no ha investigado. 
ROCÍO BERENGUER SÁNCHEZ, FÁTIMA LLAMAS SALGUERO Y VERÓNICA LÓPEZ FERNÁNDEZ RELACIÓN ENTRE CREATIVIDAD Y LATERALIDAD EN EDUCACIÓN INFANTIL

TABLA 1

Datos de porcentaje para la definición de lateralidad $(\mathrm{N}=60)$

\begin{tabular}{|l|c|c|}
\hline \multicolumn{1}{|c|}{ DEFINICIÓN LATERALIDAD } & FRECUENCIA & PORCENTAJE \\
\hline Lateralidad definida & 45 & $75 \%$ \\
\hline Lateralidad no definida & 15 & $25 \%$ \\
\hline
\end{tabular}

Tabla 2

Datos estadísticos medios de creatividad del grupo con lateralidad definida

\begin{tabular}{|l|c|c|c|c|c|}
\hline $\begin{array}{l}\text { GRUPO LATERALIDAD } \\
\text { DEFINIDA }\end{array}$ & ORIGINALIDAD & ELABORACIÓN & FLUIDEZ & FLEXIBILIDAD & CREATIVIDAD \\
\hline Media & 92,50 & 25,34 & 27,1 & 14,7 & 158,66 \\
\hline Desviación típica & 27,75 & 11,25 & 6,12 & 4,41 & 39,86 \\
\hline
\end{tabular}

TABLA 3

Datos estadísticos medios del grupo con lateralidad no definida

\begin{tabular}{|l|c|c|c|c|c|}
\hline $\begin{array}{l}\text { GRUPO LATERALIDAD } \\
\text { NO DEFINIDA }\end{array}$ & ORIGINALIDAD & ELABORACIÓN & FLUIDEZ & FLEXIBILIDAD & CREATIVIDAD \\
\hline Media & 66,26 & 14,53 & 16,63 & 14,66 & 112,08 \\
\hline Desviación típica & 27,40 & 7,64 & 7,38 & 4,40 & 43,28 \\
\hline
\end{tabular}

TABLA 4

Datos de porcentaje de variación (CV) en los dos grupos

\begin{tabular}{|l|c|c|c|c|c|}
\hline $\begin{array}{l}\text { LATERALIDAD } \\
\text { DEFINIDA }\end{array}$ & $\begin{array}{c}\text { ORIGINALIDAD } \\
30 \%\end{array}$ & $\begin{array}{c}\text { ELABORACIÓN } \\
44 \%\end{array}$ & $\begin{array}{c}\text { FLUIDEZ } \\
23 \%\end{array}$ & $\begin{array}{c}\text { FLEXIBILIDAD } \\
30 \%\end{array}$ & $\begin{array}{c}\text { CREATIVIDAD } \\
25 \%\end{array}$ \\
\hline $\begin{array}{l}\text { Lateralidad } \\
\text { no definida }\end{array}$ & $41 \%$ & $52 \%$ & $44 \%$ & $30 \%$ & $39 \%$ \\
\hline
\end{tabular}

Las Tablas 2 y 3 muestran datos medios de los componentes de la creatividad, superiores para el grupo con lateralidad definida, así como creatividad total superior (Tabla 2), frente a medias inferiores y creatividad total inferior para el grupo con lateralidad No definida (Tabla 3). Los dos grupos tienen una desviación típica alta, superior en fluidez $(7,38)$ y creatividad total $(43,28)$ para el grupo con lateralidad no definida.

En la Tabla 4 vemos el coeficiente de variación para los dos grupos. De las dos series de datos, el grupo con lateralidad definida tiene menor coeficiente, por lo que sus datos (en cada componente de la creatividad y en la creatividad total) se alejan menos de la media.

En cambio, el grupo con lateralidad no definida tiene mayores coeficientes de variación, por lo que su dispersión es mayor. 
Además, si el CV es mayor del 30\% la media no es representativa del conjunto de datos y sería conveniente, en un futuro, usar otra medida de centralización, porque para el grupo con lateralidad no definida los componentes originalidad, elaboración y fluidez superan ese 30\%.

\section{CONClusiones}

El estudio confirma el objetivo general de la investigación: el grupo con lateralidad definida obtiene mayores puntuaciones medias en cada componente de creatividad que el grupo con lateralidad no definida, así como una mayor creatividad (Tablas 2 y 3 ).

Para el grupo con lateralidad definida el CV se cumple para todos los componentes de creatividad excepto para elaboración. Sin embargo, para el grupo de lateralidad no definida el CV sólo se cumple para el componente flexibilidad, para los demás componentes la media no es representativa del conjunto de datos. Los resultados reafirman, a nivel neuropsicológico, la idea de que los niños con lateralidad no definida tienen peor rendimiento en las medidas de creatividad.

Por otro lado, el grupo con lateralidad definida obtiene mayor rendimiento en todos los componentes de creatividad, confirmando, por tanto, la corriente de pensamiento de la Asociación Norteamericana de Psicología de Washington que afirma tener evidencias de relación entre la lateralidad zurda y la creatividad intelectual.

\section{REFERENCIAS BIBLIOGRÁFICAS}

Aluni, R. y Penagos, J. (2000) Preguntas más frecuentes sobre creatividad. Revista Psicología [versión electrónica].

Bogousslavsky, J. y Bollery, F. (eds.) (2006). Neurological Disorders in Famous Artists. Boller: Karger.

Dadamia, M. (2001). Educación y creatividad. Encuentro en el nuevo milenio. Buenos Aires: Magisterio del Río de la Plata.

DeGraff, J. (1995). Creativity at work. Management Series. University of Michigan Business Scholl.

Dorsch, F. (1985). Diccionario de psicología. Barcelona: Herder.

Enciclopedia de Psicopedagogía. Pedagogía y Psicología (1998). Océano.

Fernando, M.; Prieto, M. D.; Fernández, C. y Sánchez, C. (2005). Inteligencia y creatividad. Electronic Journal of Research in Educational Psychology (Ed.). Universidad de Almería, España, 21-49.

Ferré, J. y Aribau, E. (2002). El desarrollo neurofuncional del niño y sus trastornos. Barcelona: Lebón.

Flanagán, J. C. (1957). The definition and measurement of ingenuity. En C. W. Taylor (Ed.). The second University or Utah research conference on the identification of creative scientific talent.

Getszels, J. W. y Jackson, P. (1962). Creativity and Inteligence: explorations ith gifted students. Nueva York: John Wiley. 

RELACIÓN ENTRE CREATIVIDAD Y LATERALIDAD EN EDUCACIÓN INFANTIL

Guillford, J. P. (1973). Characteristic of creativity. Springfield: Illinois State office of the superintendent of Public Instruction.

Guillford, J. P. (1976). Factores que favorecen y factores que obstaculizan la creatividad. Salamanca: Anaya.

Llamas, J. (2003). Tándem. [Versión electrónica]. Revista Tándems: 12.

Martín Lobo, P. (2003). La lectura. Procesos neuropsicológicos del aprendizaje, dificultades, programas de intervención y estudio de casos. Barcelona: Lebón.

Martín Lobo, M. P. (2012). Lateralidad y rendimiento escolar. La Rioja: UNIR.

Martín Lobo, M. P.; García-Castellón, C.; Rodríguez, I. y Vallejo, C. (2011). Test de lateralidad de las pruebas neuropsicológicas. Instituto de Neuropsicología y Educación. Madrid: Fomento.

Montañes, P. (1999). Avances en Psiquiatría Biológica, vol. 10. Colombia: Clínica de Memoria.

Montañes, P. (2001). Cerebro, Arte y Creatividad. Bogotá: Guadalupe.

Mumford, M. D. (2000). Managing creative people: Strategies and tactics for innovation. [Versión electrónica]. Human resource management review: 10.

Orborn, A. (1953). Applied Imagination. Scribner.

Portellano, J. A. (2005). Introducción a la neuropsicología. Madrid: McGraw-Hill.

Puente, A. (1999). El cerebro creador. Alianza Editorial.

Rigal, R. (1987). Motricidad humana: fundamentos y aplicaciones pedagógicas. Madrid: Editorial Pila Teleña S. A.

Regard, M. y Landis, T. (1988). Beauty may differ in each Half of the eye of the beholder. Beauty and the Brain. Biological aspect of aesthetics, vol. I. Rentschler, B. Herzberger and D. Epstein. Birkháuser Verlag: 243-256, Boston.

Sternberg, R. J. y Lubart, T. I. (1995). Defying the crowd: cultivating creativity in a culture of conformity. New York: Free Press.

Thurstone, L. (1959). The measurement of values. University of Chicago Press.

Torrance, E. P. (1974). The Torrance test of creative thinking: Norms-technical manual. Bensenville, IL: Scholastic Testing Service. 